\title{
Saúde mental no contexto de isolamento social
}

\author{
Mental health in the context of social isolation \\ La salud mental en el contexto del aislamiento social
}

\author{
Cleide Correia de Oliveira \\ ORCID: https://orcid.org/0000-0001-8135-449X \\ Universidade Regional do Cariri, Brasil \\ E-mail: cleide.correia @urca.br \\ Joaquim Rangel Lucio da Penha \\ ORCID: https://orcid.org/0000-0003-0325-3495 \\ Secretaria Municipal de Educação, Brasil \\ E-mail: rangel.lucio10@gmail.com \\ Helvis Eduardo Oliveira da Silva \\ ORCID: https://orcid.org/0000-0001-5152-5024 \\ Universidade Regional do Cariri, Brasil \\ E-mail: helviseduardo@gmail.com \\ Lorena Pinheiro Costa \\ ORCID: https://orcid.org/0000-0001-7419-8353 \\ Universidade Regional do Cariri, Brasil \\ E-mail; lory81312006@gmail.com \\ Ana Hirlene de Brito Correia Oliveira \\ ORCID: https://orcid.org/0000-0001-5134-6739 \\ Universidade Regional do Cariri, Brasil \\ E-mail: anahirlene@yahoo.com.br \\ José'Hercules Gomes da Filho \\ ORCID: https://orcid.org/0000-0003-0864-4049 \\ Universidade Regional do Cariri, Brasil \\ E-mail: jhercules05gomesrf@gmail.com
}

\begin{abstract}
Resumo
Objetivo: Identificar os graus de depressão durante a pandemia do Coronavírus COVID-19 e rastrear o impacto do distanciamento social relacionado e/ou não os indícios de depressão. Metodologia: Um estudo do tipo exploratório com abordagem quantitativa desenvolvido durante o período de agosto à dezembro de 2020, com uma população de 104 participantes entre estudantes da graduação e profissionais da saúde, com a faixa etária entre 16 a 50 anos. Foram utilizadas três variáveis: sexo, idade e estado civil, sendo analisadas quanto ao nível e predominância de Transtorno Depressivo desenvolvido durante o isolamento social. A coleta de dados utilizanda a adaptação do Questionário Patient Health Questionnaire-9 (PHQ-9), validado para o Brasil. Resultados: O sexo feminino apresentou Transtorno Depressivo Moderado (16\%), seguido por Moderamente grave (11,5\%), e em contrapartida (12,5\%) não apresentaram transtornos. Já o sexo masculino apresentou o Transtorno Depressivo Moderado (12,5\%). A idade com maiores números de casos foi de 21 a 30 anos, com Transtorno Depressivo Moderado (18,3\%), Transtorno Depressivo moderadamente grave (15\%). Quanto às atividades desenvolvidas durante o isolamento social em sua maioria responderam: brincar com os filhos, assistir séries, escrever artigos, entre outras. Com relação aos sentimentos dos participantes do estudo desenvolvidos no enfrentamento à pandemia, os participantes poderiam escolher mais de uma opção, sendo: insegurança, sobrecarregados, triste, estressados, medo, dentre outras. Conclusão: Evidenciou-se frequência elevada de sentimentos de isolamento, ansiedade e tristeza, medo, estresse no período do estudo como também os transtornos depressivos na população.
\end{abstract}

Palavras-chave: Saúde mental; Isolamento social; Depressão.

\begin{abstract}
Objective: To identify the levels of depression during the pandemic of the Coronavirus COVID-19 and to track the impact of social detachment and / or not during the period of the pandemic related to the signs of depression. Methodology: This is an exploratory study with an approach with a quantitative approach developed during the period from August to December 2020 with a population of 104 participants, including undergraduate students and health professionals, aged between 16 and 50 years old. Three variables were used: sex, age, marital status, being analyzed for the level and predominance of Depressive Disorder developed during social isolation. Data collection using the adaptation of the Questionnaire Patient Health Questionnaire-9 (PHQ-9), validated for Brazil. Results: Females presented Moderate Depressive Disorder (16\%), followed by Moderately Severe $(11.5 \%)$ on the other hand (12.5\%) did not present any disorders. The male gender, on the other hand, presented Moderate Depressive Disorder (12.5\%).
\end{abstract}


The age with the highest number of cases was 21 to 30 years, with Moderate Depressive Disorder (18.3\%), Moderately severe Depressive Disorder (15\%). As for the activities developed during social isolation, most responded: playing with their children, watching series, writing articles, among others. Regarding the feelings of study participants developed in coping with the pandemic, participants could choose more than one option, namely: insecurity, overloaded, sad, stressed, fear, among others. Conclusion: There was a high frequency of feelings of isolation, anxiety and sadness, fear, stress during the study period, as well as depressive disorders in the population.

Keywords: Mental health; Social isolation; Depression.

\section{Resumen}

Objetivo: Identificar los niveles de depresión durante la pandemia del Coronavirus COVID-19 y rastrear el impacto del desapego social y / o no durante el período de la pandemia relacionado con los signos de depresión. Metodología: Se trata de un estudio exploratorio con abordaje con abordaje cuantitativo desarrollado durante el período de agosto a diciembre de 2020 con una población de 104 participantes, entre estudiantes de pregrado y profesionales de la salud, con edades comprendidas entre los 16 y los 50 años. Se utilizaron tres variables: sexo, edad, estado civil y se analizaron el nivel y predominio del trastorno depresivo desarrollado durante el aislamiento social. Recolección de datos mediante la adaptación del Cuestionario de Salud del Paciente-9 (PHQ-9), validado para Brasil. Resultados: Las mujeres presentaron Trastorno Depresivo Moderado (16\%), seguido de Moderadamente Severo (11,5\%) en cambio $(12,5 \%)$ no presentaron ningún trastorno. El sexo masculino, en cambio, presentó Trastorno Depresivo Moderado (12,5\%). La edad con mayor número de casos fue de 21 a 30 años, con Trastorno Depresivo Moderado (18,3\%), Trastorno Depresivo Moderadamente Severo (15\%). En cuanto a las actividades desarrolladas durante el aislamiento social, la mayoría respondió: jugar con sus hijos, ver series, escribir artículos, entre otras. Con respecto a los sentimientos que los participantes del estudio desarrollaron al enfrentar la pandemia, los participantes pudieron elegir más de una opción, a saber: inseguridad, exceso de trabajo, tristeza, estrés, miedo, entre otras. Conclusión: Hubo una alta frecuencia de sentimientos de aislamiento, ansiedad y tristeza, miedo, estrés durante el período de estudio, así como trastornos depresivos en la población.

Palabras clave: Salud mental; Aislamiento social; Depresión.

\section{Introdução}

O mundo vem enfrentado desde o início do ano de 2020, uma grave crise sanitária mundial com a pandemia devido à infecção pelo novo coronavírus denominado Severe Acute Respiratory Syndrome Coronavirus 2 (SARS-CoV-2, Síndrome Respiratório Aguda Grave 2), que provoca a Coronavirus Disease 2019 (COVID-19), detectado na China em dezembro de 2019 (Organização Pan-Americana de Saúde [OPAS], 2020). Foi declarada pandemia em 12 de março de 2020 pela Organização Mundial da Saúde (OMS) (Álvarez, 2020).

A Pandemia do novo COVID-19, apontada em mais de 180 países ao redor do mundo, com um avanço de grandes proporções da contaminação da doença, os vários governos vêm adotando diversas estratégias, com o objetivo de diminuir o ritmo da progressão da doença (Kraemer et al., 2020). A sua forma de transmissão, é de pessoa para pessoa, por gotículas de saliva, espirro, tosse de secreção nasal, acompanhado por contato pela boca, nariz ou olhos, ou até mesmo, por meio de objetos e superfícies contaminadas (WHO, 2020).

A pandemia tem afligindo o mundo desde o primeiro trimestre de 2020 pelo agente patogênico coronavírus (COVID19), tem transformado de várias maneiras os aspectos da nossa vida cotidiana, sobretudo o sistema educacional. Trata-se de um período desafiador para todos nós, sejam gestores, docentes, discentes e famílias, que em um horizonte por vezes incerto, como o que estamos vivenciando, necessitamos aprender táticas de adaptação, ação e análise crítica do cenário posto. Essas medidas têm causado impactos na vida da população em diversos setores, incluindo no sistema educacional (Pinto, 2020).

O cenário mundial no enfrentamento da pandemia adotou-se como medida não-farmacológica o distanciamento e isolamento social, como sendo estratégias de controle da disseminação da contaminação na população pelo distanciamento físico e redução da mobilidade. Sabe-se que essas são as medidas possíveis no momento, porém não se deve perder de vista que o distanciamento social certamente poderá ter impactos negativos em diferentes níveis, a saber: de forma particular, nas pessoas; de forma ampliada, em seus diferentes contextos de desenvolvimento tais como: familiar, comunidade local, cidades, estados e países (Liang, 2020). 
Conforme Shan (2021), as medidas atuais de distanciamento social em vigência, tais como, o isolamento físico e os sofrimentos mentais tornaram-se um importante problema de saúde pública. Diante do exposto ressaltando que as consequências desse isolamento sobre a saúde mental passam a ser motivo de grande preocupação (Sousa et al., 2020; Fiorillo, 2020).

O Isolamento Social (IS) é adotado em casos extremos, conceitualmente, quando as pessoas mudam suas rotinas, não podem sair de suas residências como uma forma de evitar a propagação do vírus. Existe a recomendação relacionadas as pessoas suspeitas de portarem o vírus que permaneçam em quarentena por quatorze dias, pois este é o período de incubação do SARS-CoV-2, ou seja, o tempo para o vírus manifestar-se no corpo do indivíduo (Oliveira, 2020).

Já o distanciamento social, tem por finalidade evitar as aglomerações e manter a distância entre as pessoas com a fim de, no mínimo, um metro e meio entre as pessoas, como também a proibição de eventos que ocasionem um grande número de indivíduos reunidos como em escolas, universidades, shows, shoppings, academias esportivas, eventos esportivos, entre outros eventos (Reis-filho \& Quinto, 2020).

A Organização Mundial de Saúde (OMS) estimou que, em 2015, 264 milhões (3,6\%) de pessoas em todo o mundo apresentavam algum transtorno de ansiedade, sendo mais comum entre as mulheres. No Brasil, cerca de 9,3\% da população é afetada por este transtorno (World Health Organization - WHO, 2017).

Diante do exposto, torna-se importante refletir para além das condições patológicas específicas causadas pela COVID19, é essencial considerar dentro desse contexto as condições de saúde mental da população diante dos múltiplos reflexos que essa pandemia tem causado, uma vez que estudos recentes apontaram mudanças significativas no quadro de saúde mental da população em âmbito mundial (ONU, 2020).

Com isso, é preciso uma atenção especial às demandas psicológicas que podem surgir em decorrência do momento atual que o mundo enfrenta, enfatizando principalmente a necessidade pela busca de um olhar especializado, no sentido de preservar o máximo possível a saúde mental (Silva et al., 2020).

As evidências emergentes mostram que a pandemia teve impactos traumáticos na saúde mental, causando aumento dos casos de ansiedade, acarretados, substancialmente, pela solidão, incerteza e ausência de contato humano (Smith \& Li, 2020). Apesar do isolamento social e a solidão serem entendidos como condições distintas, as duas estão correlacionadas com mortalidade prematura, depressão, doença cardiovascular e declínio cognitivo (Smith \& Li, 2020). Pessoas colocadas em quarentena em surtos anteriores de doenças infecciosas relataram resultados adversos de saúde mental após o período de isolamento (Hossain et al., 2020).

A Pandemia do COVID-19, nos remete a muitas reflexões: da importância do papel social da universidade, da relevância do trabalho docente, das desigualdades sociais presentes dentro de um mesmo espaço institucional, do quanto é importante a relação interpessoal, o papel da tecnologia na vida contemporânea, etc. Frente ao exposto, esta investigação parte da seguinte questão de pesquisa: qual o nível depressão entre uma população entre diversos setores da sociedade em distanciamento social preventivo obrigatório de uma cidade do interior do interior do estado do Ceará? Quais fatores podem estar envolvidos os níveis de depressão entre dessa população em estudo?

Nessa perspectiva, percebe-se que essa problemática atinge e inquieta a comunidade universitária, prejudica sua qualidade de vida, altera rotinas familiares e desafia para a elaboração de estratégias de enfrentamento, bem como para a busca de mecanismos que propiciem melhorar a capacidade física e mental para essa "guerra" (biológica, social, psicológica e humana) que está longe de ter seu fim e que muito contribui para o aumento do estresse dos docentes e discentes.

Considerando o contexto atual da pandemia da COVID-19, tendo em vista os poucos artigos publicados relacionados aos discentes de ensino superior e de suas capacidades de resiliência, verificou-se a necessidade desse estudo, pois as 
adversidades sempre estarão presentes tanto na vida acadêmica, pessoal e profissional, e a resiliência é um ponto chave que precisa ser trabalhado.

O estudo tem como objetivos: Rastrear a o impacto do distanciamento social e/ou não durante o período da pandemia da COVID-19 relacionado os indícios de depressão.

\section{Metodologia}

Estudo com delineamento exploratório com abordagem quantitativa. A pesquisa tem como propósito proporcionar maior familiaridade com o problema, com vistas a torná-lo mais explícito ou a construir hipóteses. Os métodos exploratórios têm como finalidade proporcionar maior familiaridade com o objeto do estudo torná-lo mais explícito ou a construir hipóteses, considerar os mais variados aspectos relativos ao fato ou fenômeno estudado. Já nos delineamentos quantitativos os resultados são apresentados em termos numéricos e, nas qualitativas, mediante descrições verbais (Gil, 2017).

\section{Participantes e local de estudo}

O estudo foi desenvolvido no período de agosto a novembro de 2020 com a população geral de de 104 participantes com maiores de 18 anos, com uma população constituídas por estudantes (n=60) e docentes de instituições de ensino de nível superior e profissionais da saúde $(n=44)$. Optou-se pela amostragem não probabilística de conveniência, que é caracterizada por ser composta de indivíduos que atendem aos critérios de inclusão e são de fácil acesso ao investigador (HULLEY, 2015).

Para a população utilizamos o critério de saturação de respostas. $\mathrm{O}$ fechamento amostral por saturação teórica é operacionalmente definido como a suspensão de inclusão de novos participantes no estudo quando os dados obtidos passam a apresentar, na visão do pesquisador, certa redundância ou repetição.

\section{Instrumentos}

O instrumento utilizado para a coleta de dados incluiu pergunta sobre dados sociodemográfico (número do questionário, data, sexo, idade, escolaridade, formação profissional) e o Questionário Patient Health Questionnaire-9 (PHQ-9). Este questionário teve suas propriedades de rastreamento validadas para a população geral brasileira em 2013 (Santos et al., 2013). A coleta de dados ocorreu publicamente pelas redes sociais WhatsApp e Instagram, no período de agosto a dezembro de 2020. Os dados foram coletados por meio de questionário on-line desenvolvido no Formulários Google, contendo questões abertas e de múltipla escolha, contendo os seguintes questionamentos relacionados as últimas duas semanas: teve interesse ou pouco prazer em fazer as coisas; se sentiu para baixo, deprimido (a) ou sem perspectiva; teve dificuldade para pegar no sono ou permanecer dormindo ou dormiu mais do que de costume; se sentiu cansado(a) ou com pouca energia: teve falta de apetite ou comeu demais: se sentiu mal consigo mesmo(a) ou achou que é um fracasso ou que decepcionou sua família ou a você mesmo; teve dificuldade para se concentrar nas coisas (como ler o jornal ou ver televisão; teve lentidão para se movimentar ou falar (a ponto das outras pessoas perceberem), ou ao contrário, esteve tão agitado(a); os sintomas anteriores lhe causaram algum tipo de dificuldade para trabalhar ou estudar ou tomar conta das coisas em casa; se sente durante esse isolamento; Quais as atividades complementares mais realizadas pelo(a) $\operatorname{Sr}($ a) nesse isolamento.

O PHQ-9 avalia a presença de sintomas depressivos por meio de uma escala do tipo Likert composta por nove perguntas categorizadas em quatro opções de respostas que variam de "não, nenhum dia" (zero pontos) até "quase todos os dias" ( 3 pontos) e um escore total que varia de zero a 27 pontos. Assim, quanto maior a pontuação, pior a gravidade dos indícios depressivos. (Santos, et al, 2013).

Ao responder o inventário, o participante seleciona a opção que melhor se ajusta à maneira como se sente no momento, com uma variação dos escores entre zero (ausência do sintoma) e três (intensidade maior do sintoma). 
Podem ser localizados na literatura em diferenciadas populações/amostras (Bhardwaj et al., 2018), O que demonstra o valor do instrumento para a prática da pesquisa, já que pode apoiar aos pesquisadores na obtenção de dados sobre os sintomas depressivos (Lam, 2018).

\section{Procedimentos e metodologia de análise}

O primeiro contato realizado através da rede social em que os pesquisadores fazem participam o Instagram da Liga de Ensino Pesquisa e Extensão em saúde Mental, grupos de Whats App de algumas disciplinas de cursos de graduação, de docentes de instituições de nível superior o que torna essencial para o primeiro e posterior contato com os demais. Encaminhado via e-mails de forma individual com convites aos que tiverem interesse em participar do estudo. Mediante a assinatura do Termo de Consentimento Livre e Esclarecido e Pós - Esclarecido, para assegurar o anonimato dos convidados do estudo. Todas as questões serão inseridas em um formulário do Google Forms. Os procedimentos éticos foram cumpridos. A pesquisa aprovada pelo Comitê de Ética em Pesquisa da Universidade Regional do Cariri (CEP/URCA), com o Parecer de $\mathrm{N}^{\circ}$ 4.070.193,

Para a análise de dados, foi utilizado o software Microsoft Office Excel $2019 \circledR$ e interpretados pela estatística descritiva com formulação de tabelas. Para fins estatísticos, utilizou-se o programa Statistical Package for the social SPSS 20.0. Foram utilizadas análises descritivas com base em amostras, de modo que as informações possam ser expandidas para o todo. O nível de significância utilizado foi de $95 \%$.

\section{Resultados e Discussão}

O estudo foram 104 participantes, sendo em sua maioria do sexo feminino, entre 16 a 50 anos que correspondiam aos mais diversos setores da sociedade, acadêmicos dos cursos de enfermagem ( $n=18$ ), medicina $(n=05)$, Biologia $(n=03)$, Engenharia civil ( $n=01)$, ensino superior em andamento sem especificar o curso $(n=15)$, totalizando $(n=42)$; já os docentes do ensino superior um total de $(n=44)$, entre os docentes temos profissionais de saúde $(n=14)$ entre eles, enfermeiros, fisioterapeutas e psicólogos; alunos do ensino médio com $(\mathrm{n}=18)$. O critério de inclusão considerado foram idade superior a 18 anos ou mais, residentes no Brasil, e que finalizaram o questionário. Foram utilizadas três variáveis: sexo, idade, estado civil sendo analisadas quanto ao nível e predominância de Transtorno Depressivo (TD) desenvolvido durante o isolamento social da pandemia da Covid-19 do ano de 2020. Diante disso, os dados foram analisados criteriosamente e cada variável foi analisada de acordo com os níveis de depressão desde a ausência da doença até o transtorno depressivo grave. A pontuação total varia de 0 a 27 e representa a soma das respostas dos nove itens.

A gravidade do quadro seria estimada conforme o seguinte: 0-4 pontos - sem depressão; 5-9 pontos - transtorno depressivo leve; 10-14 pontos - transtorno depressivo moderado; 15-19 pontos - transtorno depressivo moderadamente grave e de 20 a 27 pontos - transtorno depressivo grave.

No Quadro 1 a seguir faz um resumo de cada variável seguidas das porcentagens referentes aos níveis de depressão desenvolvidas pelos participantes dessa pesquisa os quais foram classificados em: sem depressão, transtorno depressivo leve, transtorno depressivo moderado, transtorno depressivo moderadamente grave e transtorno depressivo grave. 
Quadro 1 - Nível do Transtorno de Depressão associado ao sexo a idade e o estado civil.

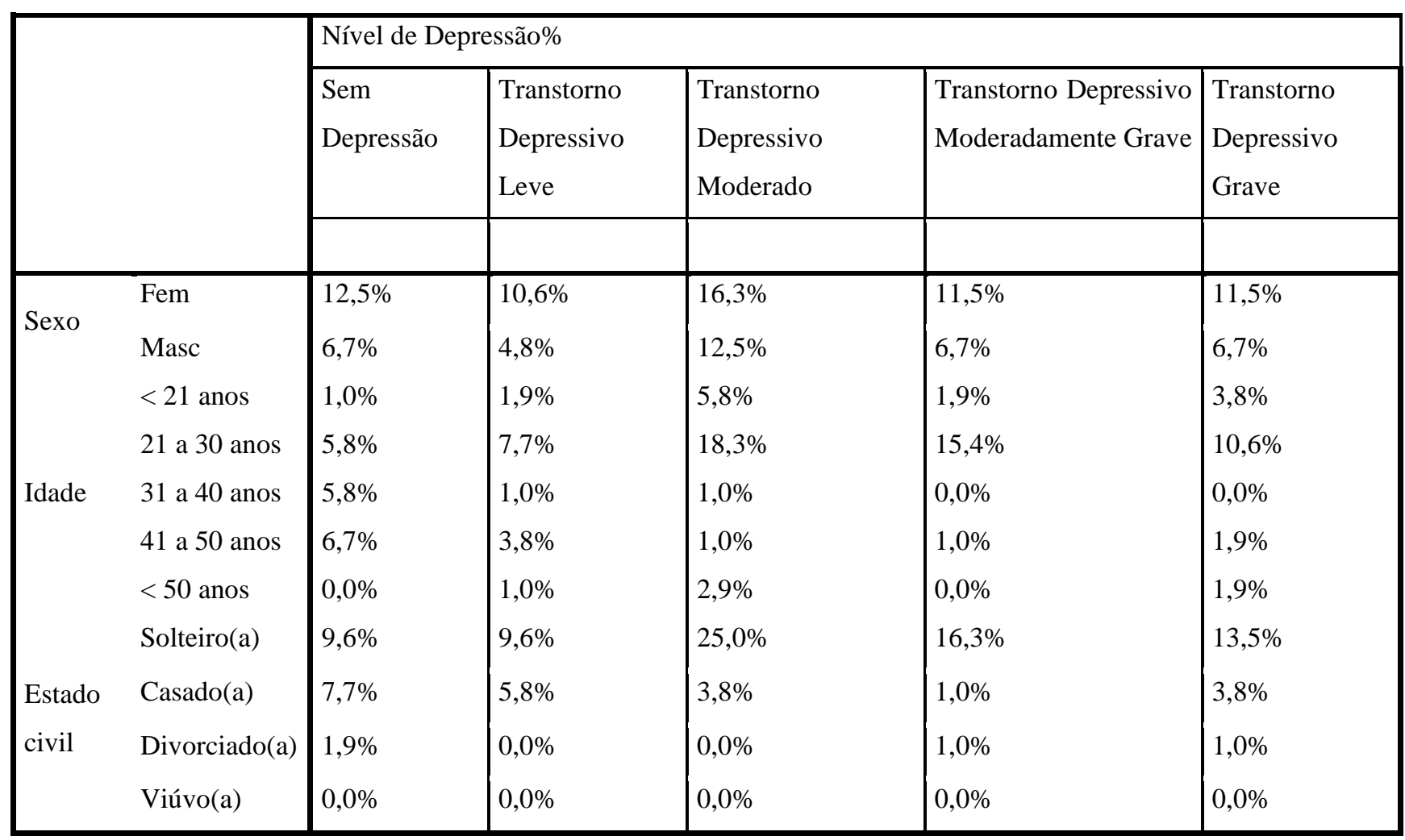

Fonte: Autores (2021).

Quando questionados sobre o impacto da pandemia e do isolamento social na sua saúde mental, os participantes declararam que houve uma influência comportamental significativa que pode contribuir como fator de risco para transtornos mentais como a depressão.

Assim, após a análise criteriosa foram elaborados gráficos específicos para cada variável, afim de detalhar melhor os achados. Iniciando com a análise dos dados referentes ao sexo e sua relação com o desenvolvimento de algum nível de Transtorno Depressivo conforme apresentando a seguir: 
Gráfico 1 - Transtornos Mentais Depressivos relacionados ao sexo.

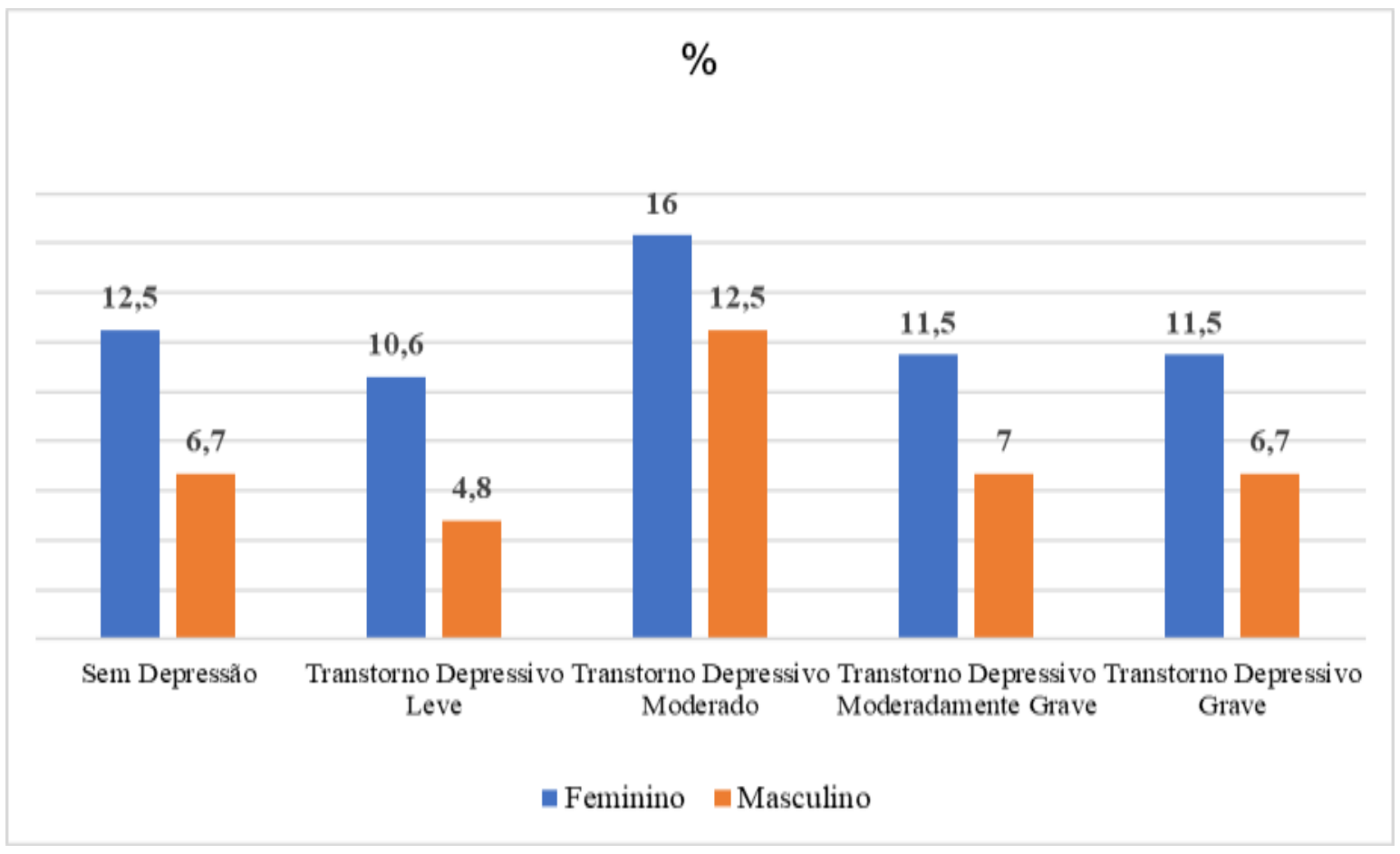

Fonte: Autores (2021).

Nesse sentido, pode-se inferir na perda de interesse ou no prazer em realizar as atividades durante o isolamento social, que são sinais característicos do transtorno depressivo, atingiram na sua grande maioria o sexo feminino representado no gráfico em mulheres com os transtorno depressivo moderado (16\%), seguido por transtornos depressivos moderamente grave $(11,5 \%)$ e transtorno depressivo grave $(11,5 \%)$, em contrapartida $(12,5 \%)$ das entrevistadas não foram observado nenhum transtorno depressivo, porém o alto índice de mulheres com depressão moderada, moderadamente grave e grave se configura como um índice preocupante frente a situação atual.

Já no sexo masculino foi observado que houve uma predominância de transtorno depressivo moderado (12,5\%), seguido de transtorno depressivo moderadamente grave (7\%), transtorno depressivo grave $(6,7 \%)$, sem depressão $(6,7 \%)$ e transtorno depressivo leve $(4,8 \%)$.Contudo, ao se fazer uma analogia entre a prevalência do transtorno entre o sexo masculino e feminino verifica-se de imediato uma disparidade significativa com predominância das mulheres como público mais acometido por depressão em seus vários estágios durante o isolamento social.

Segundo Ali et al. (2020), apontou que as mulheres, durante uma pesquisa realizada em Bangladesh, apresentaram na sua grande maioria um alto índice de estresse durante o período de isolamento social em seu país acarretando no comprometimento direto da sua saúde mental e bem-estar em comparação com os homens. Além disso, ainda segundo essa pesquisa, as mulheres encontravam-se como maioria entre os casos de depressão e $(52,7 \%)$ adquiriram algum tipo de adoecimento mental durante isolamento no seu pais, em contrapartida os homens foi de $(48,9 \%$.).

Diante dessa realidade diversos estudos de caráter epidemiológico corroboram com a informação de que o transtorno depressivo é uma realidade que atinge duas vezes mais as mulheres do que os homens. Fato esse justificado por estudos que têm buscado os fatores explicativos para essa realidade e apontam como pontos importantes as diferenças hormonais e fisiológicas das mulheres, a baixa renda, as questões culturais, bem como a postura frente a situações estressoras no seu dia a dia como causa para essa prevalência. (Gonçalves et al., 2018). 
Além disso, alguns estudos apontaram como fatores de risco para o comprometimento da saúde mental das mulheres durante a pandemia: morar em regiões que apresentem o maior número de casos e consequente mortalidade pela Covid-19, escassez em atividades físicas e lazer, desemprego, ser adulta jovem, história pregressa de ansiedade e depressão (Souza et al. 2020; Souza et al., 2020).

Gráfico 2 - Transtornos Mentais Depressivos relacionados ao estado civil

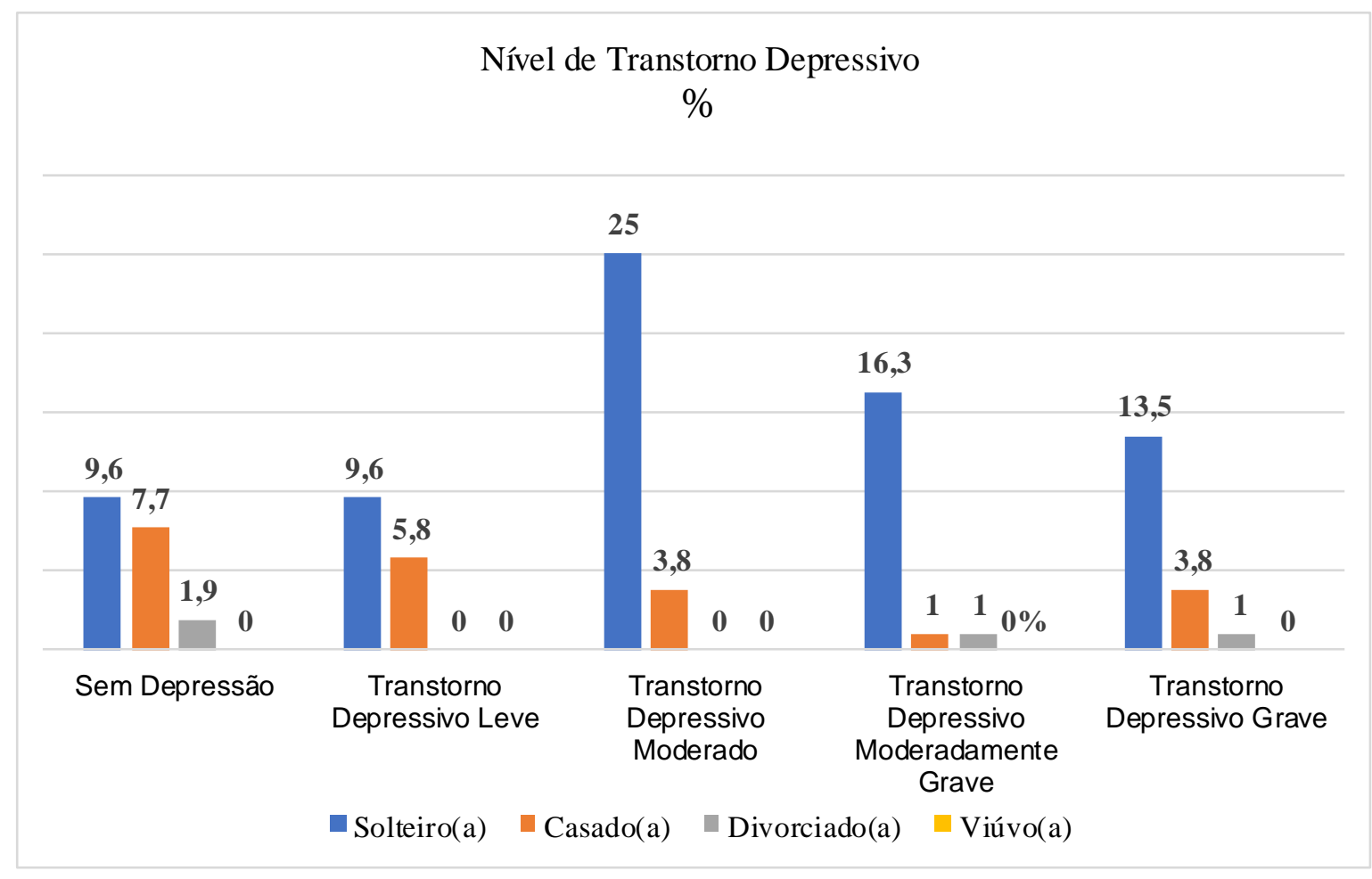

Fonte: Autores (2021).

Nessa perspectiva, outro parâmetro usado nessa pesquisa foi o estado civil, como torna-se evidente no gráfico (02), o qual foi analisado de acordo com sua influência frente ao desenvolvimento do transtorno depressivo ou até mesmo o seu agravo. Verificou- se que dentre os participantes solteiros(as), casados(as), divorciados(as) ou viúvos (as) houve uma prevalência significativa de solteiros acometidos com algum nível de depressão, dentre os participantes solteiros houve uma prevalência de transtorno depressivo moderado (25\%), o que representa uma estatística preocupante, seguida por solteiros com transtorno depressivo moderamente grave (16,3\%) transtorno depressivo grave $(13,5 \%)$, transtorno depressivo leve $(9,6 \%)$.

Em contrapartida, ser casado (a), divorciado (a), viúvo(a) apresentam impacto bem menor frente aos participantes solteiros dessa pesquisa com destaque para os participantes casados(as) que apresentaram um índice importante que foram os participantes que que não desenvolveram nenhum nível de depressão (7,7\%).

Assim, um estudo que avaliou os índices de depressão entre os idosos durante a pandemia elucidou que o não ser casado auxilia ao indivíduo viver sozinho promovendo a solidão, com destaque para o aumento considerável durante o isolamento social, contribuindo para o desenvolvimento de sintomas depressivos. Além disso, a solidão leva esses pacientes a possuírem menos animo para interagir com outras pessoas, a realizar atividades físicas prejudicando de forma direta no bem-estar psicológico durante os tempos pandêmicos (Ávila et al.,2021). 
No que tange ao risco de desenvolver transtornos mentais menores há evidencias que ser mais jovem, mulher, ter um eventual diagnóstico prévio de adoecimento mental na família, não está envolvido diretamente como trabalhador da saúde, perca de emprego durante a quarentena são fatores direto que contribuíram para prejuízos na saúde mental da população (Duarte et al.,2020).

As taxas de aumento dos Transtornos Mentais, dentro do contexto da Covid-19, mostraram que o impacto das informações recebidas diariamente pelas mídias e os altos índices de apreensão decorrentes do adoecimento de algum membro da família diagnosticados com a doença foram fatores que contribuíram diretamente para o aumento considerável dos níveis de estresse, ansiedade, depressão e angustia da população (Wang et al.,2020).

Além disso, o fato de ser profissional da saúde contribui na diminuição de $40 \%$ das chances de desenvolver algum transtorno mental menor. Mesmo que ainda estejam em constante contato direto com a doença, em comparação com a grande massa da população, existem fatores que contribuem significativamente para esse diminuição como: o maior acesso dos profissionais de saúde a assistência especializada, maior conhecimento frente as medidas de controle, proteção e tratamento da Covid-19. Em contrapartida cabe ressaltar que os profissionais de saúde em sua grande parcela estavam em isolamento social, fato esse que pode contribuir eficazmente para a atenuação das chances de serem infectados e consequentemente diminuir a ansiedade relacionada ao medo da doença e ao risco de adquirir algum transtorno mental (Duarte et al.,2020).

Gráfico 3 - Níveis de Transtornos Depressivos relacionados a idade.

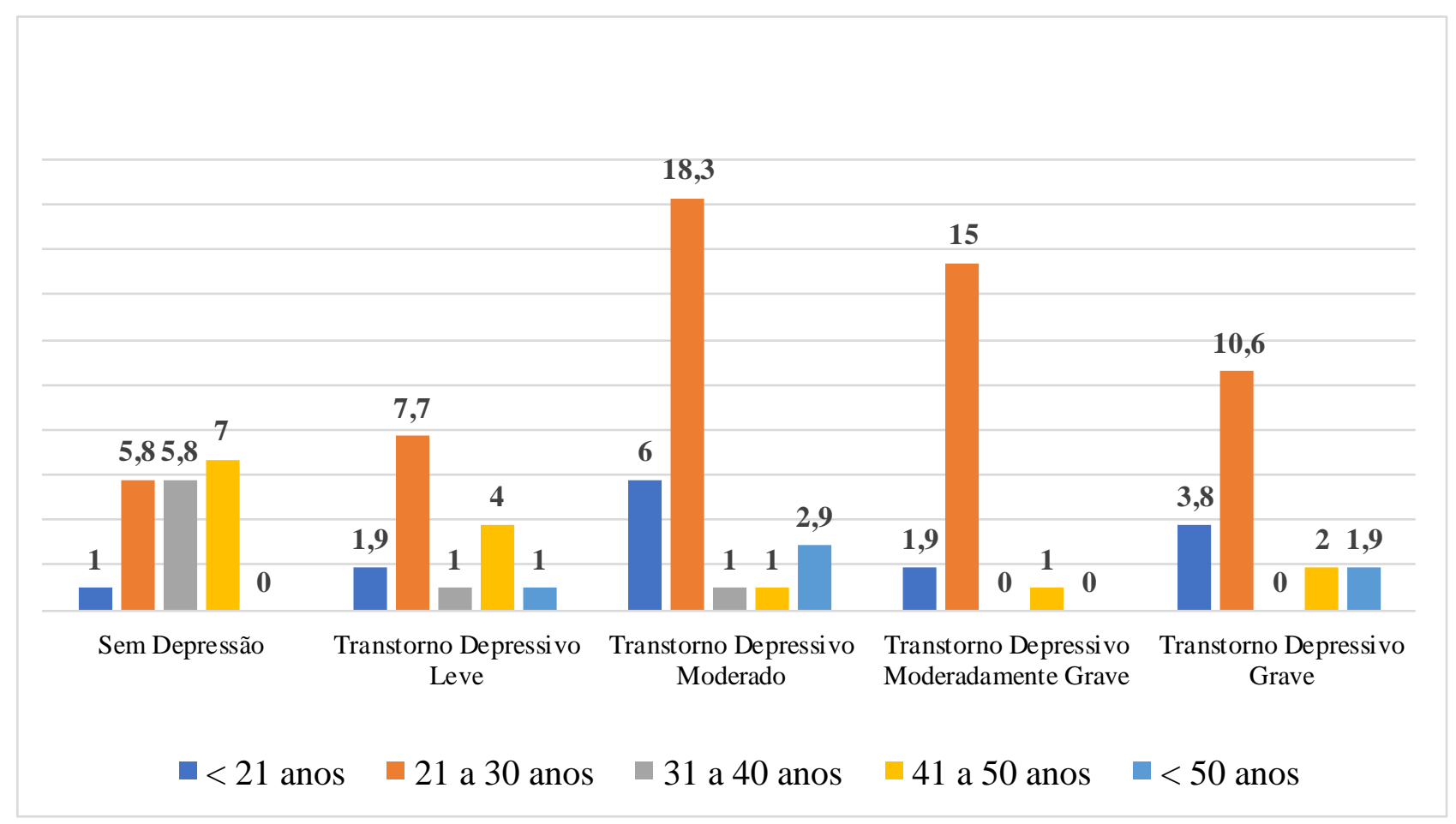

Fonte: Autores (2021).

Nesse sentido, outra variável de extrema importância na avaliação desse estudo é a faixa etária. Foi constatado que dentro da faixa etária dos participantes dessa pesquisa houve uma predominância alarmante no desenvolvimento de transtornos depressivos entre os indivíduos com idades de 21 a 30 anos, sendo uma das faixas mais acometidas e expressos no Gráfico 3, acima da seguinte forma: transtorno depressivo moderado (18,3\%), 
transtorno depressivo moderadamente grave (15\%), transtorno depressivo grave $(10,6 \%)$ e transtorno depressivo leve $(7,7 \%)$, representando um achado importante nesse estudo.

Essa realidade é corroborada por algumas pesquisas que indicaram que os adultos jovens, entre a faixa etária de 19 a 29 anos, foram os mais acometidos frente ao surgimento de sintomas negativos na sua saúde mental durante a pandemia da Covid-19 sendo iniciados por sintomas como insônia ou até mesmo agravamento da ansiedade. Fatores que contribuíram para um surgimento de transtornos depressivos nessa faixa etária está na necessidade destes estarem online por mais tempo por causa de atividades acadêmicas, escolares ou trabalhos, excesso de atividades on-line como jogos, redes sociais são fatores que podem ter contribuído para uma prevalência de transtornos mentais nessa faixa etária (Barros et al., 2020).

A depressão é uma doença complexa, que pode levar a complicações que atingem inúmeras esferas da vida de um indivíduo. No cenário do estudante de Medicina, isto inclui o desempenho acadêmico e a relação médico-paciente (Cybulski, et al., 2017).

Ademais, as mudanças abruptas na vida diária da população, gerada pela pandemia da COVID-19, e o consequente isolamento social como medida direta para combater o aumento dos casos em todo o mundo acarretou em diversos efeitos negativos na saúde da população, como por exemplo, o impacto emocional e o desenvolvimento de patologias como transtornos de ansiedade e depressão que representam situações preocupantes e que não foram traduzidas em mais demanda para a assistência em saúde. Outra influência negativa foi o aumento no consumo de álcool pelos mais jovens durante a quarentena e o desânimo que afligiu diretamente aqueles que perderam seus empregos durante esse período auxiliando em uma mudança significativa no bem-estar da população (Perman et al.,2020).

Quando questionados sobre as atividades realizadas durante o isolamento social obrigatório durante a pandemia em sua maioria responderam: jogar em casa com os filhos $(n=15)$; ler $(n=15)$, estudar $(n=04)$, exercício físicos $(n=15)$, organizar a casa $(n=13)$, aula online $(n=10)$, dormir, repousar $(n=10)$, escrever artigos $(n=05)$, assistir filmes séries $(\mathrm{n}=08)$, e brincar com os filhos $(\mathrm{n}=04)$.

Com relação aos sentimentos dos participantes do estudo desenvolvidos no enfrentamento à COVID-19, os participantes poderiam escolher mais de uma opção, em sentimento foi desenvolvido em relação à COVID-19. Sendo o seguinte: Insegurança, $(n=03)$, sentem-se sobrecarregados diante ao enfrentamento da Covid 19, $(n=10)$, triste $(n=10)$, estressados $(n \S 10)$, perdido $(n=09)$, medo $(n=06)$, tédio $(n=05)$, impotente $(n=09)$, um lixo $(n=04)$, preso $(n=03)$, cansado ( $n=03)$, com medo $(n=06)$, até o momento bem $(n=09)$, com dores de cabeça $(n=05)$, ansioso $(n=05)$, ineficaz ( $\mathrm{n}=03)$, saudades da família(n=03), sufocado $(\mathrm{n}=02)$.

Corroborando com os nossos estudos Junior et al., (2020) descreve que muitos sentimentos são manifestados durante a vivência de uma pandemia, as incertezas e o medo vão desencadeando demais emoções que em maioria são negativas. Brooks et al, (2020), afirma que evidências recentes sugerem que as pessoas que são mantidas em isolamento e quarentena experimentam níveis significativos de ansiedade, raiva, confusão e estresse.

Além disso, o isolamento social durante a pandemia resulta na exposição do sofrimento em grande parte da população, causados pelas preocupações como: problemas relacionados a economia e a explosão de notícias na mídia, bem como outras questões relacionadas a realidade de cada uma das pessoas que mantem relações cotidianas. Tais fatores podem ocasionar a manifestação ou o agravamento de sintomas psicológicos como a ansiedade, o estresse e a depressão (Orrnell et al., 2020).

O distanciamento social contribuiu para um impacto negativo na saúde mental e nos estilos de vida dos brasileiros, com aumento do sentimento ansiedade, isolamento, tristeza ou depressão. Além disso, observou-se aumento 
do uso de bebidas alcoólicas e tabaco, redução da prática de atividade física e aumento do consumo de alimentos não saudáveis (Malta et al., 2020).

De acordo com Brooks et al. (2020) em seu estudo sobre impactos psicológicos e psiquiátricos devido ao coronavírus, estas causas do adoecimento mental geram desconfortos de ordem mental como cefaleia, alterações no sono, humor deprimido, aumento da agressividade, dificuldade na tomada de decisão, alteração da atenção e da memória, além de limitações na concentração.

O isolamento social pode gerar muitos efeitos negativos, como nas emoções, pensamentos e no corpo, e com essas medidas, de fechamento de escolas, universidades, estabelecimentos e de contatos físicos rotineiras, o medo, ansiedade em pessoas intensificam cada vez mais e sendo principal causador de medo, ansiedade, incerteza pelo futuro, tristeza, solidão, irritabilidade e angústia (Pereira et al., 2020).

Mesmo não havendo exposição direta à infecção, pode-se vivenciar ansiedade, raiva, desesperança, medo, insônia, sensação de desamparo e solidão (Ahmeda et al., 2020; Nabuco et al., 2020; Oliveira 2020; Afonso, 2020).

A restrição espacial, a falta de lazer, a ansiedade, o estresse e o tédio, estão associados a um maior consumo alimentar. Os efeitos negativos associados do distanciamento social poderão ser observados a curto, médio e longo prazo na saúde individual e coletiva (Malta et al., 2020).

Além dos impactos biológicos e econômicos, o surgimento súbito de uma doença sempre representa uma ameaça à saúde mental, podendo ocasionar um amplo e variável espectro de manifestações de adoecimento mental, que afeta inclusive, pessoas consideradas saudáveis (Ahmeda et al., 2020; Nabuco et al., 2020; Oliveira 2020; Afonso, 2020).

\section{Conclusão}

Evidenciou-se frequência elevada de sentimentos de isolamento, ansiedade, tristeza, medo, estresse, cansaço, sentimentos de aprisionamento, impotência, entre outros no período do estudo. Esses achados são preocupantes e podem resultar em danos à saúde e implicações psicológicas. Entretanto, o estudo apontou que essa medida pode resultar em consequências psicossociais da população com elevado índice na população em estudo de Transtorno Depressivo Moderado (18,3\%), Transtorno Depressivo Moderadamente grave (15\%), Transtorno Depressivo Grave (10,6\%) e Transtorno Depressivo Leve (7,7\%), representando um achado importante nesse estudo.

O distanciamento social conforme Malta et al. (2020), constitui-se medida essencial de proteção à vida, de redução de exposição, reduzindo casos e consequentemente a morbimortalidade. Portanto, esforços imediatos devem ser empregados, em todos os níveis e pelas mais diversas áreas de conhecimento, a fim de minimizar resultados ainda mais negativos na saúde mental da população. Em contrapartida, o isolamento social tem provocado um aumento significativo de transtornos depressivos, como o explanado no presente estudo, havendo uma necessidade terapêutica voltada para a prevenção desses tipos de transtornos durante a pandemia da covid-19.

Assim, o presente estudo contribuiu para um impacto negativo na saúde mental e nos estilos de vida dos brasileiros, com aumento do sentimento ansiedade, isolamento, tristeza ou depressão. Além das medidas para o enfrentamento e contenção da pandemia, é preciso garantir à população uma assistência em saúde mental, com ações que minimizem o sofrimento mental ao longo desse período de crise, bem como suporte de uma rede psicossocial de acesso e cuidado em transtornos desde leve e severo. É preciso trabalhar estratégias que visem à promoção da saúde mental, tanto a curto quanto a médio e logo prazo, na tentativa de reduzir ou prevenir problemas psiquiátricos e psicológicos (Faro et al., 2020).

Vale ressaltar algumas dificuldades encontradas na realização do estudo, tais como, demais referências envolvendo as temáticas, referências de estudos semelhantes em outros grupos de discentes e docentes de outras IES e 
regiões geográficas; bem como a limitação em ampliar uma maior divulgação do instrumento de coleta de dados e da pesquisa devido a falta de inclusão digital de parte significativa dos discentes que se encontram em suas cidades de referência familiar/moradia de origem, tais como, por falta de acesso à internet e por não possuir aparelho tecnológico habilitado para o uso adequado.

A realização desse estudo evidenciou a importância de se ter um cuidado voltado para a prevenção e promoção em saúde mental, redução de transtornos mentais como a depressão, durante o período de isolamento, visto que, de acordo com os resultados apontados, houve um aumento significativo de transtornos depressivos leves e moderados. Ressaltando ainda, a importância de efetivação das políticas públicas de saúde mental de prevenção e promoção em saúde mental, além do estudo possibilitar avaliar esse aumento, o qual poderá servir como fundamento para estratégias de desenvolvimento de medidas terapêuticas que visem um tratamento adequado para esse público, bem como métodos de promoção da saúde mental para os indivíduos mais atingidos.

\section{Referências}

Ahmed, M. Z., Ahmed, O., Aibao, Z., Hanbin, S., Siyu, L., \& Ahmad, A. (2020). Epidemic of COVID-19 in China and associated Psychological Problems. Asian journal of psychiatry, 51, 102092

Afonso P. O impacto da pandemia COVID-19 na saúde mental. Act Med Port. 33(5):356-7.

Ali, M., Ahsan, G. U., Khan, R., Khan, H. R., \& Hossain, A. (2020). Immediate impact of stay-at-home orders to control COVID-19 transmission on mental well-being in Bangladeshi adults: Patterns, Explanations, and future directions. BMC research notes, 13(1), 1-7.

Barros, M. B. D. A., Lima, M. G., Malta, D. C., Szwarcwald, C. L., Azevedo, R. C. S. D., Romero, D., \& Gracie, R. (2020). Relato de tristeza/depressão, nervosismo/ansiedade e problemas de sono na população adulta brasileira durante a pandemia de COVID-19. Epidemiologia e Serviços de Saúde, 29, e2020427.

Bhardwaj, M., Price, J., Landry, M., Harvey, P., \& Hensel, J. M. (2018). Association between severity of depression and cardiac risk factors among women referred to a cardiac rehabilitation and prevention clinic. Journal of cardiopulmonary rehabilitation and prevention, $38(5)$, 291-296. 10.1097/HCR.0000000000000311.

Brooks, S. K., Webster, R. K., Smith, L. E., Woodland, L., Wessely, S., Greenberg, N., \& Rubin, G. J. (2020). The psychological impact of quarantine and how to reduce it: rapid review of the evidence. The lancet, 395(10227), 912-920.

Cybulski, C. A., \& Mansani, F. P. (2017). Análise da Depressão, dos Fatores de Risco para Sintomas Depressivos e do Uso de Antidepressivos entre Acadêmicos do Curso de Medicina da Universidade Estadual de Ponta Grossa. Revista Brasileira de Educação Médica, 41(1), 92-101.

Duarte, M. D. L. C., Silva, D. G. D., \& Bagatini, M. M. C. (2021). Enfermagem e saúde mental: uma reflexão em meio à pandemia de coronavírus. Revista Gaúcha de Enfermagem, 42(SPE).

Faro, A., Bahiano, M. D. A., Nakano, T. D. C., Reis, C., Silva, B. F. P. D., \& Vitti, L. S. (2020). COVID-19 e saúde mental: a emergência do cuidado. Estudos de Psicologia (Campinas), 37.

Fiorillo, A., Sampogna, G., Giallonardo, V., Del Vecchio, V., Luciano, M., Albert, U., \& Volpe, U. (2020). Effects of the lockdown on the mental health of the general population during the COVID-19 pandemic in Italy: Results from the COMET collaborative network. European Psychiatry, 63(1).

Gil, A. C. (2017). Pós-Graduação-Metodologia-Como Elaborar Projetos de Pesquisa-Cap 2.

Gonçalves, A. M. C., Maria, T. B. T., Jairo, R. A. G., Claudia, S. L., Gulnar, A. S.,Carmen,J. G., Kristiane, C. D. D. (2018). Prevalência de depressão e fatores associados em mulheres atendidas pela estratégia de saúde da família. Artigo original - j. Bras. Psiquiatr. 67 (2).

HULLEY, Stephen et al. Delineando a pesquisa clínica. (4a ed.), ArtMed, 2015.

Hossain, M. M., Sultana, A., \& Purohit, N. (2020). Mental health outcomes of quarantine and isolation for infection prevention: a systematic umbrella review of the global evidence. 4178/epih.e2020038.

Lam, R. W. (2018). Depression. (3a ed.), Oxford University Press.

Liang, T. (2020). Zhejiang University School of Medicine. Handbook of COVID-19: prevention and treatment. UNESCO.

Malta, D. C., Gomes, C. S., Szwarcwald, C. L., de Azevedo Barros, M. B., da Silva, A. G., Prates, E. J. S., \& da Silva, D. R. P. (2020). Distanciamento social, sentimento de tristeza e estilos de vida da população brasileira durante a pandemia de COVID-19. 10.1590/SciELOPreprints.1371.

Nabuco, G., Pires de Oliveira, M. H. P., \& Afonso, M. P. D. (2020). O impacto da pandemia pela COVID-19 na saúde mental: qual é o papel da Atenção Primária à Saúde? Revista Brasileira De Medicina De Família E Comunidade, 15(42), 2532. 
Oliveira, L. D. Espaço e Economia: Novos Caminhos, Novas Tensões. Espaço e Economia. 2020. 1(17), 1-13. Oliveira, L. D. D. (2012). Espaço e Economia: Novos Caminhos, Novas Tensões. Espaço e Economia. Revista brasileira de geografia econômica, (1).

Organização das Nações Unidas (ONU). (2020). ONU diz que medo não pode viralizar após pandemia de novo coronavírus.https://news.un.org/pt/story/2020/03/1707031.

Ornell, F., et al. Pandemia de medo e COVID-19: impacto na saúde mental e possíveis estratégicas. Revista debates in psychiatry, 2020

Pahayahay, A., \& Khalili-Mahani, N. (2020). What Media Helps, What Media Hurts: A Mixed Methods Survey Study of Coping with COVID-19 Using the Media Repertoire Framework and the Appraisal Theory of Stress. Journal of medical Internet research, 22(8), e20186.

Pereira-Ávila, F. M. V., Lam, S. C., Goulart, M. D. C., Góes, F. G. B., Pereira-Caldeira, N. M. V., \& Gir, E. (2021). Fatores Associados Aos Sintomas De Depressão Entre Idosos Durante A Pandemia Da Covid-19. Texto \& Contexto-Enfermagem, 30.

Pinto F. R., Silva J. S., Nogueira T. V., \& Ferreira T. C. (2014). Resiliência em discentes de administração, poridade, religiosidade e gênero. Revista da faculdade de administração e economia. Ceará; 5(2): 141-62.

Reis-Filho, J. A., \& Quinto, D. (2020). COVID-19, Afastamento social, pesca artesanal e segurança alimentar: como esses temas estão relacionados e quão importante é a soberania dos trabalhadores da pesca diante do cenário distópico.

Santos, I. S., Tavares, B. F., Munhoz, T. N., Almeida, L. S. P. D., Silva, N. T. B. D., Tams, B. D., \& Matijasevich, A. (2013). Sensibilidade e especificidade do Patient Health Questionnaire-9 (PHQ-9) entre adultos da população geral. Cadernos de Saúde Pública, 29, $1533-1543$.

Silva, H. G. N., dos Santos, L. E. S., \& de Oliveira, A. K. S. (2020). Efeitos da pandemia do novo Coronavírus na saúde mental de indivíduos e coletividades/Effects of the new Coronavirus pandemic on the mental health of individuals and communities. Journal of Nursing and Health, 10(4).

Smith, B. J., \& Lim, M. H. (2020). How the COVID-19 pandemic is focusing attention on loneliness and social isolation. Public Health Res Pract, 30(2), 3022008. 10.17061/phrp3022008.

Shan, W. S. Y., Zhang, D., Shan, S. R. W., Kei, Y. B. H., Chung R. Y., \& Man Wong C. K. Impact of COVID-19 on loneliness, mental health, and health service utilisation: A prospective cohort study of older adults with multimorbidity in primary care. British Journal of General Practice [Internet].

Sousa, M. N. A. D., Estrela, Y. D. C. A., \& Bezerra, A. L. D. (2020). Perfil epidemiológico de casos de coronavírus no estado da Paraíba utilizando o Boletim Epidemiológico Local.

Souza, A. S. R., Souza, G. F. D. A., \& Praciano, G. D. A. F. (2020). A saúde mental das mulheres em tempos da COVID-19. Revista Brasileira de Saúde Materno Infantil, 20(3), 659-661.

Wang, C., Pan, R., Wan, X., Tan, Y., Xu, L., Ho, C. S., \& Ho, R. C. (2020). Immediate psychological responses and associated factors during the initial stage of the 2019 coronavirus disease (COVID-19) epidemic among the general population in China. International journal of environmental research and public health, 17(5), 1729 .

WHO, World Health Organization. (2020). Mental health and psychosocial considerations during the COVID-19 outbreak.

WHO, World Health Organization.(2020). Mental health and psychosocial considerations during the COVID-19 outbreak. 